\title{
Laparoscopic Anterior Resection with Transvaginal Specimen Extraction (TVSE) for Colorectal Cancer and Concomitant Total Hysterectomy and Bilateral Salpingo-Oophrectomy (THBSO): A Technical Description
}

\author{
Chun-Han Nigel Tan, MBBS, MRCS (Edin) ${ }^{1,2}$, Choon-Seng Chong, MBBS, M Med (Surgery), FRCSEd (Gen \\ Surg) ${ }^{1,2}$, Yoke-Fai Fong, MBBS, MRCOG (UK), M Med (O\&G), FRANZCOG (Aust/NZ), FAMS ${ }^{3}$, Shakina Rauff, \\ MBBS, MRCOG (UK), M Med (O\&G), FAMS ${ }^{4}$, and Ker-Kan Tan, MBBS, M Med (Surgery), FRCSEd (Gen \\ Surg) ${ }^{1,2}$
}

${ }^{1}$ Division of Colorectal Surgery, Department of Surgery, National University Health System, Singapore, Singapore; ${ }^{2}$ Department of Surgery, Yong Loo Lin School of Medicine, National University of Singapore, Singapore, Singapore; ${ }^{3}$ Division of Benign Gynaecology and Minimally Invasive Surgery, Department of Obstetrics and Gynaecology, National University Health System, Singapore, Singapore; ${ }^{4}$ Division of Reproductive Endocrinology and Infertility and Minimally Invasive Surgery, Department of Obstetrics and Gynaecology, National University Health System, Singapore, Singapore

\begin{abstract}
Background. Natural orifice specimen extraction (NOSE) has evolved to circumvent the need for a specimen extraction site. Transvaginal specimen extraction (TVSE) for colorectal disease has been shown to be safe and feasible in selected cases. ${ }^{1,2}$ We describe our technique of TVSE in a case of laparoscopic ultra-low anterior resection (ULAR) with defunctioning ileostomy (DI) with a concomitant total hysterectomy and bilateral salpingo-oophrectomy (THBSO).

Methods and Results. A 74-year-old Chinese female was diagnosed with a mid-rectal cancer following colonoscopic evaluation for a change in bowel habits. Preoperative magnetic resonance imaging (MRI) suggested T2N0 disease and the patient was recommended for upfront surgery following multidisciplinary discussion. Computed tomography (CT) scan confirmed a $4.3 \times 3.4 \mathrm{~cm}$ right adnexal cystic lesion, without enhancing septations or soft tissue component. No metastatic disease was identified. The patient underwent a
\end{abstract}

Electronic supplementary material The online version of this article (doi:10.1245/s10434-016-5478-7) contains supplementary material, which is available to authorized users.

(C) Society of Surgical Oncology 2016

First Received: 14 February 2016;

Published Online: 5 August 2016

K.-K. Tan, MBBS, M Med (Surgery), FRCSEd (Gen Surg)

e-mail: ker_kan_tan@nuhs.edu.sg laparoscopic ULAR with DI and THBSO with TVSE; operative time was $469 \mathrm{~min}$. The specimen showed a $2.5 \mathrm{~cm}$ mid-rectal tumour. Histology revealed a pT3N1a moderately differentiated adenocarcinoma of the mid rectum, with 1 of 20 lymph nodes involved by metastatic carcinoma. The quality of the total mesorectal excision (TME) was good, with no breach in the mesorectal fascia. The distal and radial margins were 1.5 and $3.0 \mathrm{~cm}$, respectively. The patient recovered well postoperatively, with minimal wound site pain, and was discharged well on postoperative day 5 .

Conclusion. TVSE is oncologically safe and feasible in certain malignant colorectal pathologies. It is an option to consider in selected cases that require a concomitant gynecological procedure.

ACKNOWLEDGMENTS The paper is not based on a previous communication to a society or meeting.

FUNDING No sources of funding were used for this research and/ or publication.

\section{REFERENCES}

1. Diana M, Perretta S, Wall J, et al. Transvaginal specimen extraction in colorectal surgery: current state of the art. Colorectal Dis. 2011;13:e104-111.

2. Kim J, Shim M, Kwun K. Laparoscopic-assisted transvaginal resection of the rectum. Dis Colon Rectum. 1996;39:582-3. 